\title{
Identification and bioinformatics analysis of pseudogenes from whole genome sequence of Phaeodactylum tricornutum
}

\author{
JI ChangMian ${ }^{1 \dagger}$, HUANG AiYou $^{2,3 \dagger}$, LIU WenLing $^{1}$, PAN GuangHua ${ }^{1} \&$ WANG GuangCe ${ }^{2^{*}}$ \\ ${ }^{1}$ Tianjin Key Laboratory of Marine Resources and Chemistry, College of Marine Science and Engineering, Tianjin University of Science and \\ Technology, Tianjin 300457, China; \\ ${ }^{2}$ Institute of Oceanology, Chinese Academy of Sciences (IOCAS), Qingdao 266071, China; \\ ${ }^{3}$ Graduate University of Chinese Academy of Sciences, Beijing 100049, China
}

Received October 10, 2011; accepted March 20, 2012; published online November 28, 2012

\begin{abstract}
Pseudogenes share sequence similarities with functional genes, but in general they have lost their protein-coding ability. The identification of pseudogenes is a very important step in genome annotation. Phaeodactylum tricornutum is a marine diatom that is rich in polyunsaturated fatty acids (PUFAs). The genome of $P$. tricornutum has been completely sequenced. To identify pseudogenes in $P$. tricornutum, we developed a pipeline to discover and characterize pseudogenes. We identified a total of 1654 'true' processed pseudogenes, 714 duplicated pseudogenes and 4729 pseudogene fragments. The results of the bioinformatics analysis indicated that the genome sequence of $P$. tricornutum contained many pseudogenes and pseudogene fragments.
\end{abstract}

Phaeodactylum tricornutum, pseudogene, pseudogene fragment, bioinformatics

Citation: Ji C M, Huang A Y, Liu W L, et al. Identification and bioinformatics analysis of pseudogenes from whole genome sequence of Phaeodactylum tricornutum. Chin Sci Bull, 2013, 58: 1010-1018, doi: 10.1007/s11434-012-5174-3

Pseudogenes are sequences that share close similarities to one or more paralogous functional genes, but in general are not expressed in the cell [1,2]. Based on their origin, pseudogenes have been divided into two categories: duplicated pseudogenes and processed pseudogenes. The former are produced by genomic DNA duplication or by the unequal exchange of chromosomes and retain the basic structure of functional genes, for example, promoters and introns [3-5]. The latter originate from the reverse transcription of mRNA that was inserted back into the genomes and processed pseudogenes rarely contain promoters or introns $[1,6]$; some of them, however, do retain a part of the poly A tail which may not have been fully degraded. The identification of pseudogenes in genomes is important for the accurate identification and annotation of the functional genes, for the evolutionary analysis of genomes and functional genes [7], and for the determination of the function of the pseudogenes [8-10]. Pseudogenes are commonly found in

\footnotetext{
$\dagger$ These authors contributed equally to this work.

* Corresponding author (email: gcwang@qdio.ac.cn)
}

the genomes of a great variety of species, having been found in E. coli [11], yeast [12], Arabidopsis [13,14], rice [15], nematodes [16], Drosophila [17], mice [18], and humans $[19,20]$. It has been predicted that the human genome encodes from 22000 to 75000 genes, about $22 \%$ of which are pseudogenes [21,22].

Plankton consists mainly of diatoms and planktonic diatoms are widely distributed in oceans, lakes, rivers and other water areas where they play a very important role in the carbon fixation and inorganic circulation cycles, particularly the silicon cycle. Nearly $20 \%$ of primary productivity is contributed by diatoms [23-26]. Diatoms have a special evolutionary position in that they originated from secondary endosymbiosis. These algae, therefore, make good material for evolutionary studies [27-31]. Phaeodactylum tricornutum is a pennate diatom that is used in the aquaculture industry as bait for shellfish and shrimp because of the abundant unsaturated fatty acids that it produces. The $33 P$. tricornutum chromosomes and the chloroplast genome have been fully sequenced [32]. Because the genome was found 
to contain many functional genes derived from animals, plants and bacteria, the $P$. tricornutum genome has been reported to be more like an animal genome than a plant genome [32]. The $P$. tricornutum genome size is $27 \mathrm{Mb}$ encoding 10402 genes [32]; approximately 400 functional genes/Mb. In contrast, there are only 7-25 functional genes/ $\mathrm{Mb}$ in the human genome $[19,20]$, much lower than in the $P$. tricornutum genome. The high functional gene density in the $P$. tricornutum genome could mean that there are only a few or no pseudogenes in the P. tricornutum genome.

In this study, we used bioinformatics methods based on C\# in the visual studio.net platform and the SQL Server platform to create a procedure to comprehensively search for pseudogenes from the P. tricornutum genome [19]. We investigated the correlations between the chromosome length, the GC content, the completeness, and the identity of pseudogenes and the corresponding functional genes. The results showed that there were many processed pseudogenes and pseudogene fragments in the $P$. tricornutum genome. These data will make a significant contribution to in-depth analyses of the $P$. tricornutum genome and its functional genes.

\section{Materials and methods}

\subsection{Source data sets}

The publicly available data that were used in this study were the genomic sequence, a functional protein data set and the cDNA data set that corresponds to the functional protein dataset. The genomic data were downloaded from the NCBI (http://www.ncbi.nlm.nih.gov/genome/) and included the sequences of the $33 P$. tricornutum chromosomes (Assembly name: ASM15095v1). The functional protein data set (Phatr2_chromosomes_geneModels_FilteredModels2_aa.fa sta.gz) and the corresponding cDNA data set (Phatr2_ chromosomes_geneModels_FilteredModels2_nt.fasta.gz) were downloaded from the U.S. Department of Energy Joint Genomes Institute (DOE-JGI) (http://genome.jgipsf.org/Phatr2/ Phatr2.download.ftp.html). The filtered models ("best") that correspond to the non-redundant protein and cDNA data sets (FilteredModels2 data) were used in this study.

\subsection{Bioinformatics methods and statistical analysis}

All the bioinformatics methods used in this paper were developed using $\mathrm{C \#}$ on the visual studio.net platform and SQL on the SQL Server platform. Statistical analysis was carried out using the numpy (http://numpy.scipy.org) and scipy (http://www.scipy.org/) modules of python. $R^{2}$ was obtained by linear regression based on the stats.linregress function of the numpy module. In this paper $R^{2}$ is defined as the correlation coefficient.

\subsection{Pseudogene identification pipeline}

The $P$. tricornutum pseudogene identification pipeline is shown in Figure 1 and each step is described as follows. (i) A six-frame translation of the entire genome was searched against the functional protein data set of $P$. tricornutum using tBlastn (main parameters: default SEG low-complexity filter parameters (12 2.2 2.5), -1E -4, -F T, -M BLOSUM62) and the Biopython (http://biopython.org/wiki/Biopython) module was used to parse the Blast results. (ii) All matches with more than $40 \%$ identity were extracted. (iii) Repetitive sequences were removed and identical sequences were merged. When the overlap of adjacent sequences was over $30 \mathrm{bp}$, the sequences were arranged in descending order according to their importance (the higher the $E$-value the lower their importance) and matching objects of low importance were removed. Sequences fulfilling the following three criteria were considered as the same candidate pseudogene (a) $|\mathrm{q} 21-\mathrm{q} 12| \leqslant \max (20,0.2 \times L)$, (b) $c 21-\mathrm{c} 12$ $\leqslant 3000$, and (c) c22-c $21 \leqslant 60$ (these parameters are the same as those that were defined previously [33]). The longest $P$. tricornutum intron was $9880 \mathrm{bp}$ and the shortest was 20 bp. In total, $99.3 \%$ of the introns were shorter than 3000 bp (the intron data are from the previously published annotated genome [32]). (iv) The coding and non-coding sequences of the matched sequences were obtained using the C\# code. (v) Sequences with $E$-values $>10^{-10}$ were removed. (vi) The functional genes and potential function genes were removed from the list of matched sequences. (vii) Redundant sequences and sequences containing repetitive and low complexity regions were also removed. (viii) Candidate pseudogenes were classified according to the sizes of gaps that they contained (gap size was obtained by the pairwise alignment between the candidate pseudogenes and the coding regions of the corresponding functional genes). If candidate pseudogene contained gaps that were longer than 20 bp (the shortest intron was $20 \mathrm{bp}$ ), it was regarded as a candidate duplicated pseudogene that contained introns; if the gaps were shorter than $20 \mathrm{bp}$, then the sequence was processed as a candidate pseudogene. (ix) The introns of a candidate duplicated pseudogene were analyzed by RepeatMasker (http://www.repeatmasker.org/). If the introns were identified as repeated sequences, then they were designated to be non-intron sequences and the candidate duplicated pseudogenes containing these sequences were considered as "disrupted" processed pseudogenes. These "disrupted" pseudogenes were regarded as being produced by the insertion of repeated sequences that occurred during the evolution of the processed pseudogenes. ( $\mathrm{x}$ ) Candidate processed pseudogenes were divided into processed pseudogenes and pseudogene fragments depending upon whether or not they spanned $>70 \%$ of the length of the corresponding functional gene. (xi) Processed pseudogenes were divided into "true" processed pseudogenes or "putative" processed pseudogenes depending on whether or not they contained an ORF disruption (frameshift and/or mutation). 


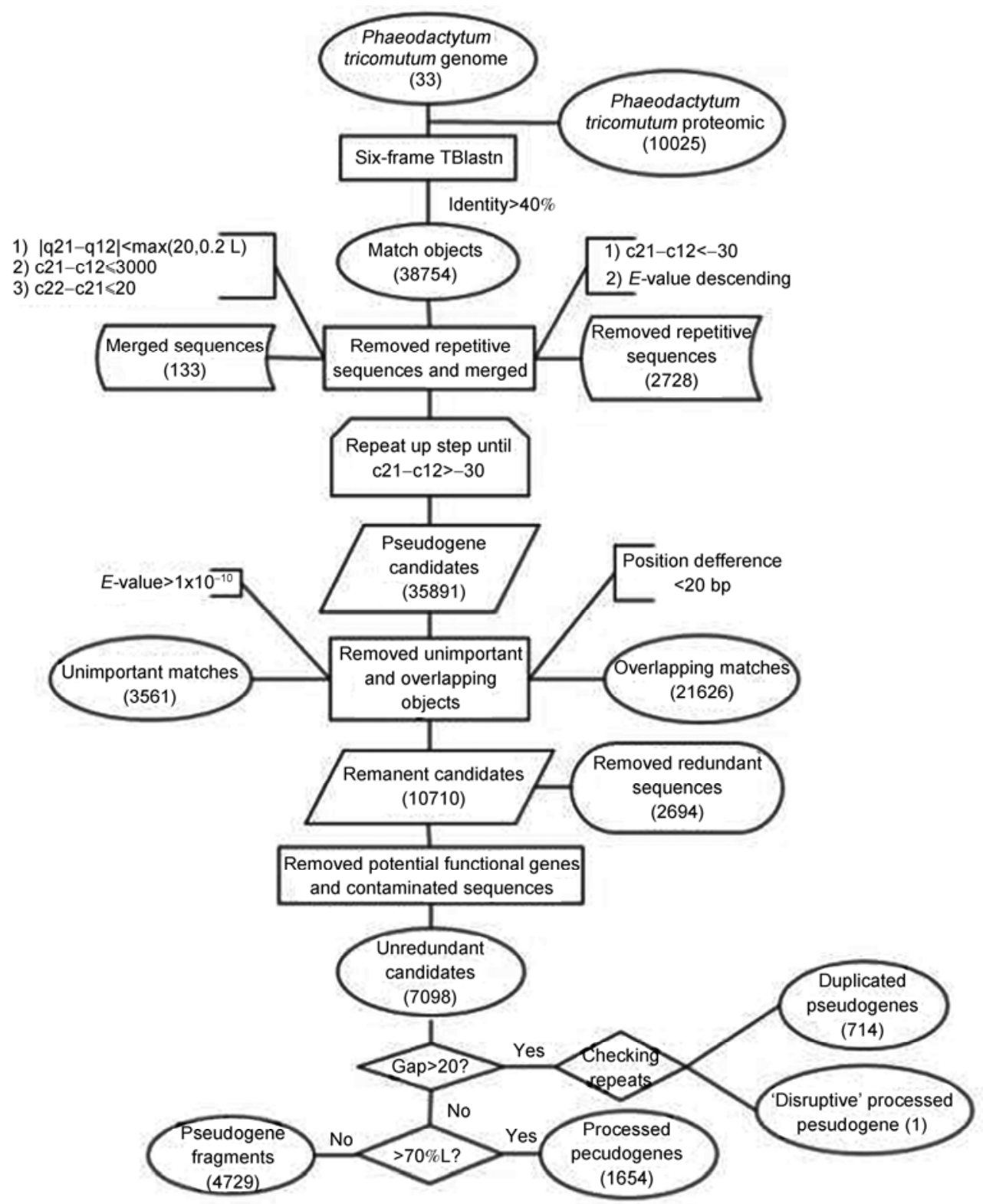

Figure 1 P. tricornutum pseudogene identification pipeline. The parameters are the same as those that have been defined previously [19]. Schematic graph showing the considerations used in merging two adjacent matches M1 and M2. (c11, c12) and (c21, c22) are chromosomal coordinates for M1 and M2; (q11, q12) and (q21, q22) are the corresponding matching regions on the query functional protein [33].

\section{Results and discussion}

\subsection{Total number and distribution of pseudogenes in $P$. tricornutum}

As shown in Figure 2, 1654 processed pseudogenes $(23.3 \%$ of the total), 714 non-processed pseudogenes (10.1\% of the total) and 4729 pseudogene fragments $(66.6 \%$ of the total) were obtained by bioinformatics analysis. The processed pseudogenes were divided into three categories: "true" processed pseudogenes, "putative" processed pseudogenes, and "disruptive" processed pseudogenes (Table 1). There were less numbers of "true" processed pseudogenes than there were "putative"; there was only one "disruptive" processed pseudogenes, indicating that there were no insertions of repeated sequences in most of the ORFs of the processed pseudogenes. These results are very different from what has been reported for other species (such as human and rice $[14,19])$ in which "disruptive" pseudogenes are commonly found.

The distribution of processed pseudogenes, pseudogene fragments and functional genes in each $P$. tricornutum chromosome was analyzed. The results revealed that the distribution of pseudogenes on each of the chromosome was not equal (Figure 3). The largest numbers of "true" processed pseudogenes and "putative" pseudogenes (115 and 35 
Table 1 Distribution of pseudogenes on each P. tricornutum chromosome

\begin{tabular}{|c|c|c|c|c|c|c|c|c|c|}
\hline \multirow{2}{*}{ Chr } & \multirow{2}{*}{ Size $(k b)$} & \multirow{2}{*}{ GC content $(\%)$} & \multirow{2}{*}{ Functional gene } & \multicolumn{3}{|c|}{ Processed pseudogene } & \multirow{2}{*}{$\begin{array}{c}\text { Pseudogene } \\
\text { fragment }\end{array}$} & \multirow{2}{*}{$\begin{array}{c}\text { Duplicated } \\
\text { pseudogene }\end{array}$} & \multirow{2}{*}{$\begin{array}{c}\text { Density } \\
\text { (per } 0.1 \mathrm{Mb}\end{array}$} \\
\hline & & & & True $^{\text {a) }}$ & Putative $^{\text {b) }}$ & Disrupted & & & \\
\hline 1 & 2535.40 & 47 & 1038 & 35 & 115 & & 403 & 66 & 5.9 \\
\hline 2 & 1497.95 & 47 & 611 & 6 & 73 & & 152 & 31 & 5.3 \\
\hline 3 & 1460.05 & 48 & 552 & 22 & 69 & & 333 & 45 & 6.2 \\
\hline 4 & 1360.15 & 48 & 538 & 12 & 45 & & 177 & 27 & 4.2 \\
\hline 5 & 1098.05 & 48 & 430 & 4 & 58 & & 168 & 21 & 5.6 \\
\hline 6 & 1035.08 & 49 & 445 & 6 & 54 & & 126 & 24 & 5.8 \\
\hline 7 & 1029.02 & 46 & 389 & 9 & 45 & 1 & 133 & 23 & 5.2 \\
\hline 8 & 1007.77 & 48 & 401 & 4 & 33 & & 154 & 13 & 3.7 \\
\hline 9 & 1002.81 & 46 & 379 & 13 & & & 168 & 33 & 1.3 \\
\hline 10 & 976.49 & 48 & 377 & 4 & 33 & & 124 & 10 & 3.8 \\
\hline 11 & 945.03 & 48 & 367 & 16 & 48 & & 167 & 19 & 6.8 \\
\hline 12 & 901.85 & 47 & 351 & 25 & 64 & & 266 & 39 & 9.9 \\
\hline 13 & 887.52 & 48 & 357 & $26(21)^{\mathrm{c})}$ & $68(1)^{c)}$ & & $201(17)^{\mathrm{c})}$ & 32 & 10.6 \\
\hline 14 & 829.36 & 48 & 316 & 14 & 29 & & 112 & 24 & 5.2 \\
\hline 15 & 814.91 & 49 & 321 & 8 & 28 & & 115 & 30 & 4.4 \\
\hline 16 & 764.23 & 48 & 291 & 29 & 44 & & 183 & 17 & 9.6 \\
\hline 17 & 703.94 & 49 & 260 & 15 & 33 & & 195 & 36 & 6.8 \\
\hline 18 & 702.47 & 49 & 259 & 11 & 63 & & 235 & 14 & 10.5 \\
\hline 19 & 690.43 & 49 & 258 & 7 & 28 & & 83 & 22 & 5.1 \\
\hline 20 & 683.01 & 49 & 260 & 11 & 41 & & 129 & 30 & 7.6 \\
\hline 21 & 662.22 & 49 & 254 & 3 & 34 & & 86 & 14 & 5.6 \\
\hline 22 & 591.34 & 48 & 236 & 5 & 20 & & 83 & 15 & 4.2 \\
\hline 23 & 512.49 & 49 & 192 & 0 & 24 & & 109 & 6 & 4.7 \\
\hline 24 & 511.74 & 48 & 158 & 4 & 19 & & 139 & 18 & 4.5 \\
\hline 25 & 497.27 & 49 & 182 & 13 & 36 & & 111 & 20 & 9.9 \\
\hline 26 & 441.23 & 47 & 141 & 11 & 38 & & 62 & 13 & 11.1 \\
\hline 27 & 404.30 & 48 & 147 & 9 & 21 & & 93 & 16 & 7.4 \\
\hline 28 & 387.58 & 47 & 167 & 4 & 7 & & 50 & 8 & 2.8 \\
\hline 29 & 384.26 & 41 & 96 & 7 & 24 & & 122 & 15 & 8.1 \\
\hline 30 & 317.21 & 49 & 104 & 4 & 13 & & 74 & 5 & 5.4 \\
\hline 31 & 258.24 & 48 & 78 & 12 & 40 & & 101 & 18 & 20.1 \\
\hline 32 & 157.05 & 40 & 44 & 1 & 8 & & 32 & 8 & 5.7 \\
\hline 33 & 87.97 & 45 & 23 & 3 & 10 & & 43 & 2 & 14.8 \\
\hline Total & 24612623 & 48 & 10022 & 353 & 1301 & 1 & 4729 & 714 & \\
\hline
\end{tabular}

a) True processed pseudogenes contain frameshift disruption; b) putative processed pseudogenes contain no frameshift disruption; c) the numbers in brackets represent the number of pseudogenes containing a polyA tail.
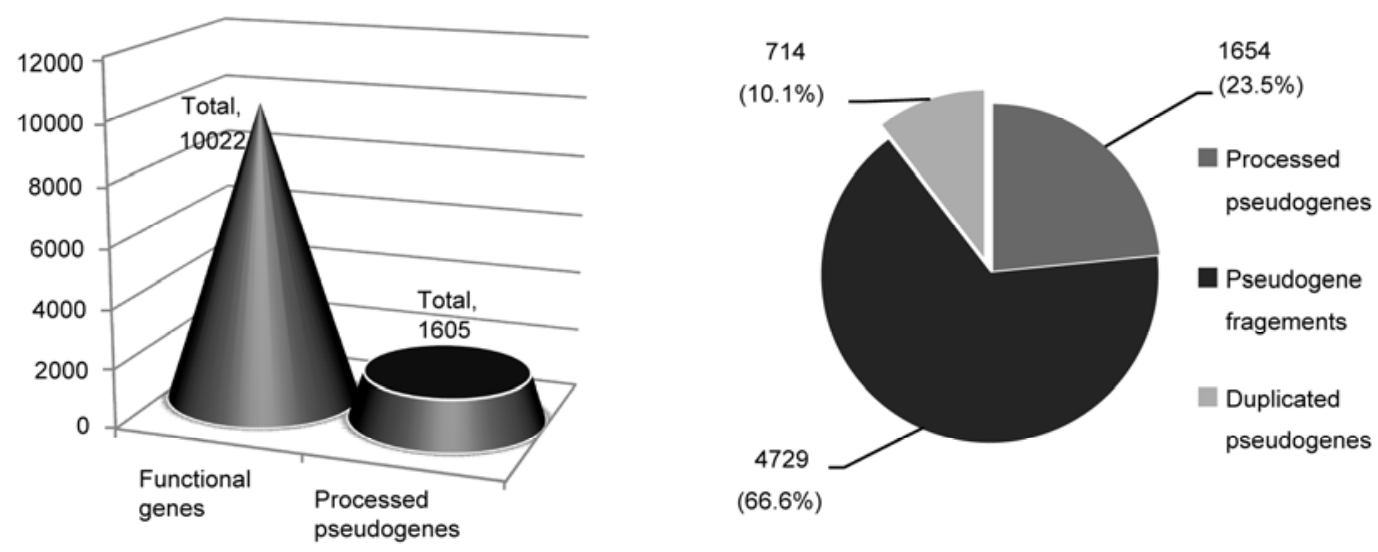

Figure 2 Statistics for the processed pseudogenes, pseudogene fragments, duplicated pseudogenes and functional genes. 


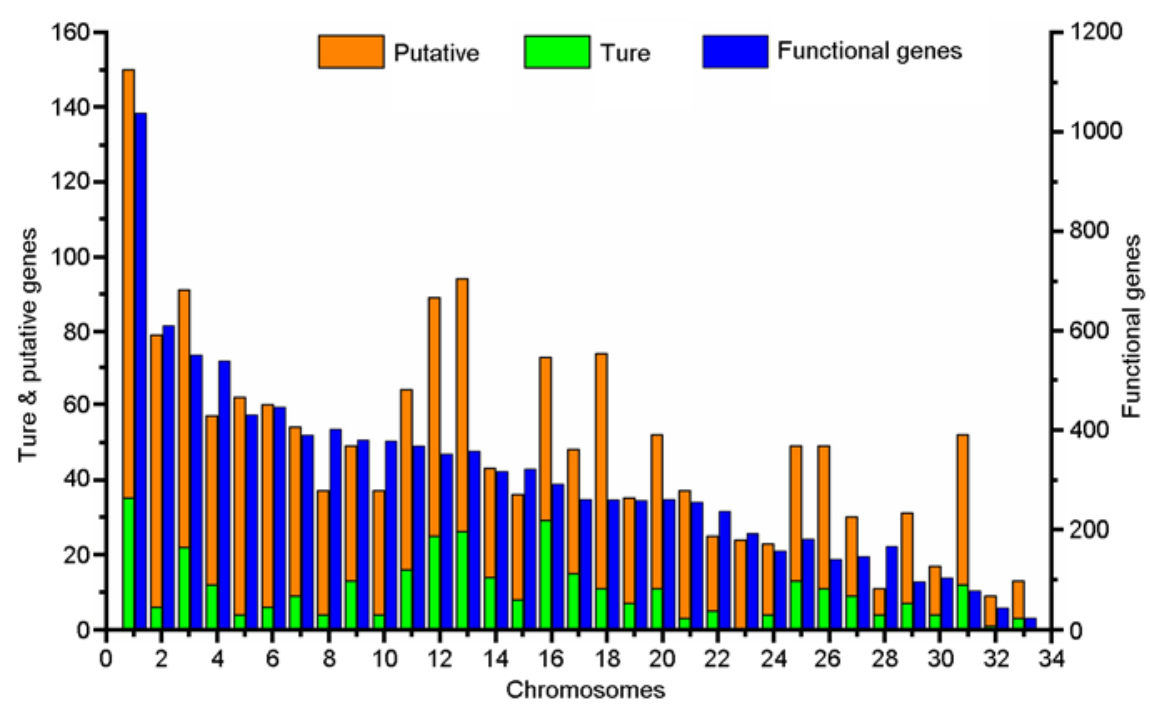

Figure 3 Distribution of "true" processed pseudogenes, "putative" processed pseudogenes, and functional genes across the different $P$. tricornutum chromosomes.

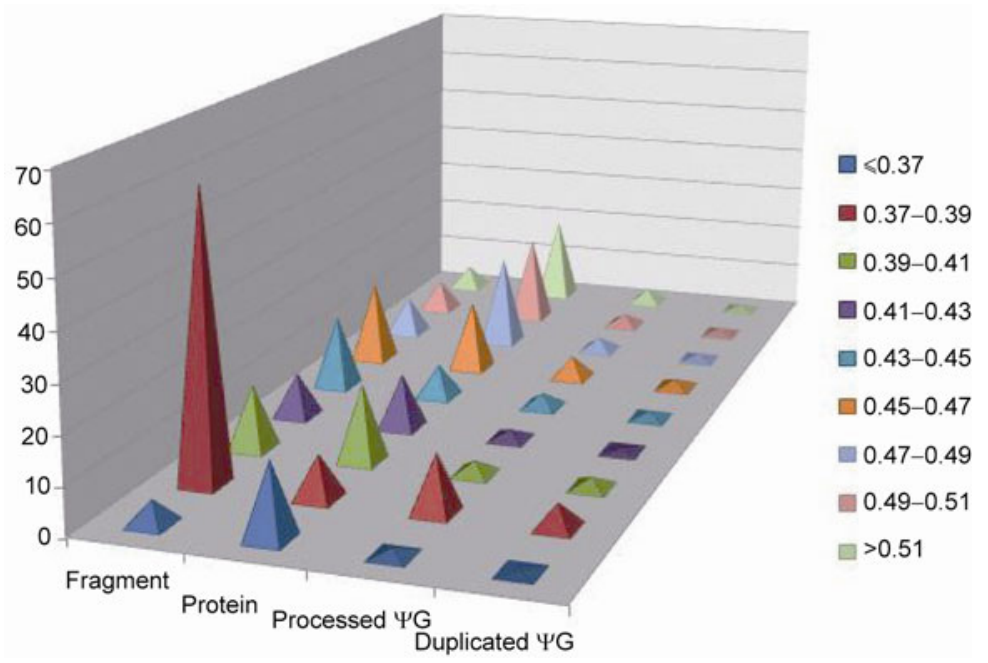

Figure 4 Distribution of pseudogene fragments, functional genes, processed pseudogenes, and duplicated pseudogenes related to the GC content of the $P$. tricornutum genome. The $X$-axis represents the sequence segments, the $Y$-axis represents the sequence densities (per $50 \mathrm{~kb}$ ), and the $Z$-axis represents the GC content. The genome sequences were divided into $50 \mathrm{~kb}$ long segments and the number of pseudogene fragments, functional genes, processed pseudogenes, and duplicated pseudogenes in each segment were calculated and related to its GC content.

respectively) were on chromosome 1 , and no "true" processed pseudogenes were found on chromosome 23. The density distribution of the processed pseudogenes and pseudogene fragments in the chromosomes were very similar; the distribution of pseudogene fragments and processed pseudogenes in regions with different GC content were also very similar (Figures S1 and 4). These results suggested that there was high correlation between the processed pseudogenes and the pseudogene fragments. Therefore, the selection of only the processed pseudogenes for further study should have no effect on the results of the analyses in this study.

Only the "true" and "putative" processed pseudogenes and the pseudogene fragments on chromosome 13 retained traces of the poly(A) tail and each of the poly(A) fragments were very short, about $15 \mathrm{bp}$ (Table 1). It has been suggested that, over time, the remaining poly(A) fragments in the processed pseudogenes will slowly degrade or even disappear $[33,34]$. Thus, some of the pseudogenes in chromosome 13 of $P$. tricornutum might have emerged later than the pseudogenes in other chromosomes.

\subsection{Correlation between chromosome length and the number of processed pseudogenes}

As shown in Figures 5 and S2, there is a linear relationship between the number of processed pseudogenes or functional genes in a chromosome and the length of chromosomes $\left(R^{2}\right.$ $=0.61$ and 0.99 respectively); that is, the longer the chromosome the greater the number of processed pseudogenes 
and functional genes that it contains. For example, there were 150 processed pseudogenes and 1038 functional genes in chromosome 1 , the longest $P$. tricornutum chromosome, while in chromosome 33, the shortest of the chromosomes, there were only 13 processed pseudogenes and 23 functional genes. The longer chromosome sequences may present more opportunity for the insertion by retrotransposition of mRNA. This conclusion is consistent with the results of Zhang et al. [19] who analyzed pseudogenes in the human genome.

\subsection{Correlation between the number of the processed pseudogenes and the GC content of the genome}

The GC content of each chromosome was calculated and then the $P$. tricornutum genome sequence was cut into 50 kb-long non-overlapping segments and the GC content and number of pseudogenes in each segment was counted. For pseudogenes that were located between two adjacent segments, the segment that contained the longer part of the pseudogene was taken to be the segment that contained that pseudogene. In addition, the distribution of the 1654 processed pseudogenes, 4729 pseudogene fragments, functional protein and duplicated pseudogenes in each segment was analyzed. The results showed that the GC content on each $P$. tricornutum chromosome was evenly distributed, and was mainly between $45 \%$ and $49 \%$ (Figure S3 and Table 1). The results in Figure 4 indicate that the distribution of the functional proteins on the genome was fairly uniform, and consequently had no correlation with the genome internal GC content; on the other hand, pseudogene fragments, processed pseudogenes and duplicated pseudogenes tended to be enriched in areas of lower GC content (37\%-41\%) (Figure 4), possibly because of a negative selection pressure that might have confronted the pseudogenes [19]. However, as the data in Figure S4 show, there was no correlation between the average GC content of a chromosome and the density of the pseudogenes (per $0.1 \mathrm{Mb}$ ) that it contained. The results shown in Figure 5 revealed that the length of the chromosome sequence did play a dominant role in its pseudogene content, indicating that in local regions of $P$. tricornutum genome, pseudogenes tended to occur in areas with lower GC content possibly as the result of negative selection pressure [19].

\subsection{Completeness of processed pseudogenes and their identity with corresponding functional gene sequences affects the number of pseudogenes}

The ratio of the lengths of the pseudogene and the corresponding functional gene was important in deciding the completeness of the pseudogene sequences. If the ratio was greater than $70 \%$, the pseudogene was considered to be a processed pseudogene; if not, it was classified as a pseudogene fragment [19]. The data in Figure 6 showed that most of the pseudogenes were almost complete after the

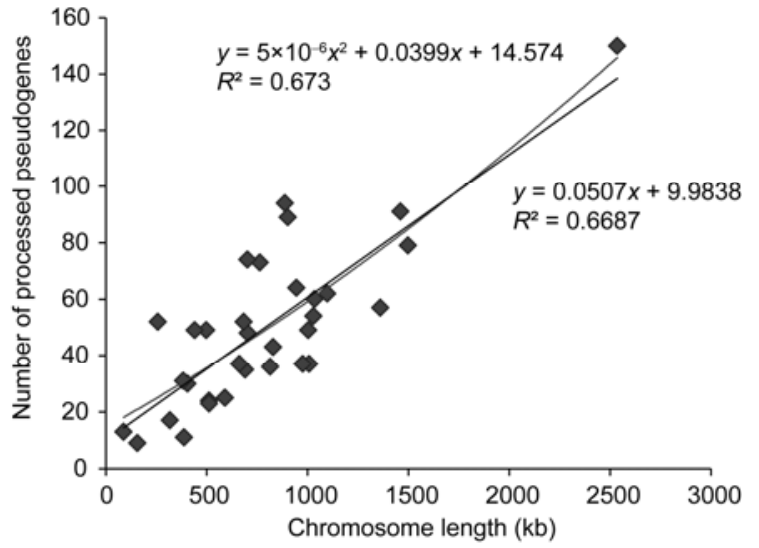

Figure 5 Correlation between chromosome length and the number of processed pseudogenes.

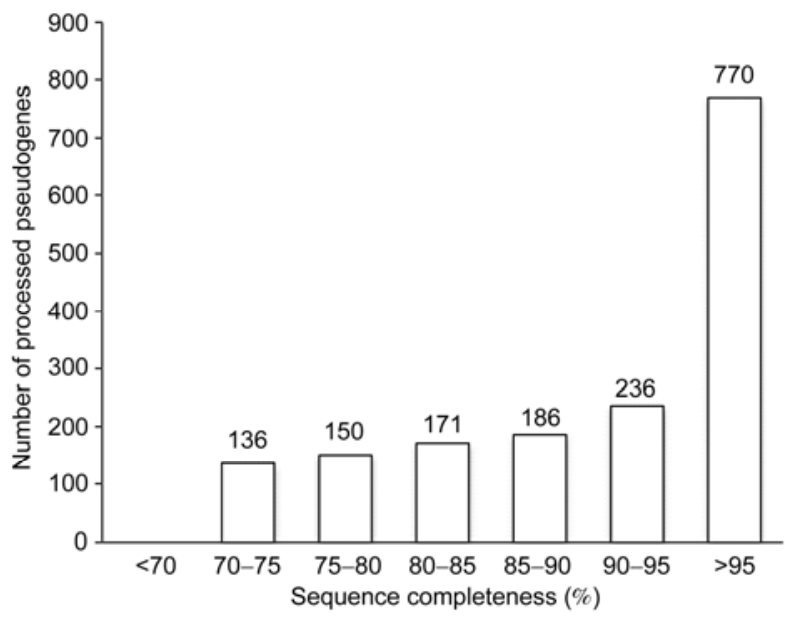

Figure 6 Correlation between the completeness of the pseudogene sequences and the number of processed pseudogenes in the P. tricornutum genome.

retrotransposition; the average completeness of the "true" processed pseudogenes was $95 \%$ and for the "putative" processed pseudogenes was 92\%. Of all the processed pseudogene sequences, $1000(61 \%)$ were more than $90 \%$ complete (Table S1).

The pseudogenes had identities that were either less than $50 \%$ or greater than $90 \%$ with their corresponding functional gene (Figure 7); this finding is very different from what was reported for the processed pseudogenes in the human genome where the number of pseudogenes tended to increase with increasing identity [19]. Figure S5 shows the relationship between the identities of the $P$. tricornutum pseudogenes and their completeness. It was found that the higher the identity of the pseudogene with the corresponding functional gene, the higher the sequence completeness $\left(R^{2}=0.72\right)$. It has been suggested that newly formed pseudogenes have a higher level of sequence completeness and identity compared to those that emerged earlier [19]. Therefore, the $P$. tricornutum pseudogenes with identities 


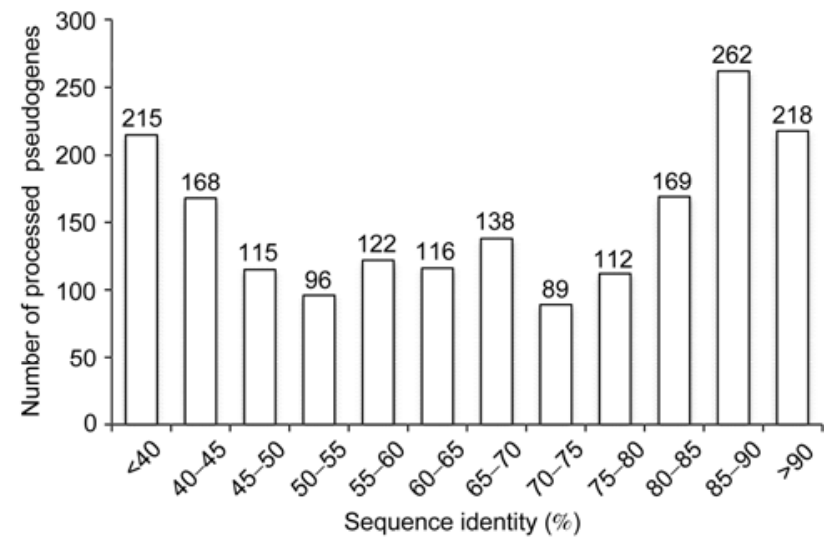

Figure 7 Correlation between the pseudogenes percentage identities with the corresponding functional genes and the number of processed pseudogenes.

greater than $90 \%$ may have formed more recently than the pseudogenes with identities less than 50\%; these pseudogenes may have formed much earlier. This suggestion implies that there may have been two active periods during which the pseudogenes of $P$. tricornutum formed. During the intervening period, the environment in which $P$. tricornutum lived may have experienced significant changes.

\subsection{Relationship between the number of processed pseudogenes or pseudogene fragments and the length of the corresponding functional genes}

When the number of processed pseudogenes within each functional gene was calculated, it was found that most of the functional genes (9101) had no corresponding processed pseudogenes; 732 functional genes had one corresponding processed pseudogene and a very small number $(77$, about $0.8 \%$ ) had more than three corresponding processed pseudogenes. A few functional genes (23) had more than 10 corresponding processed pseudogenes. Similar results were found for the number of pseudogene fragments that corresponded to functional genes. This trend can be illustrated by plotting the number of pseudogene fragments against the logarithm of the corresponding number of functional proteins (Figure 8).

Table S2 lists the corresponding homologous proteins that had more than 10 processed pseudogenes. Because the annotation of the $P$. tricornutum genome is incomplete, most of these homologous proteins were annotated as "predicted protein"; this made it difficult to functionally classify the homologous proteins that had the largest numbers of pseudogenes.

To analyze the relationship between the lengths of the functional proteins and the number of corresponding processed pseudogenes, the numbers of processed pseudogenes and pseudogene fragments in consecutive 200 amino acid long windows (with no overlap between two consecutive window positions) were calculated. The results showed that

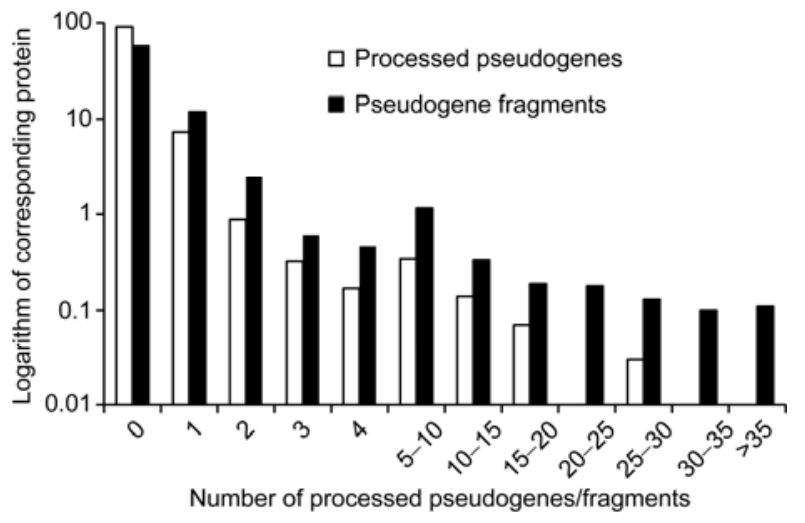

Figure 8 Number of proteins that have associated homologous processed pseudogenes or pseudogene fragments.

the number of processed pseudogenes and pseudogene fragments tended to decrease as the length of the functional genes increased (Figure S6). Thus, it would appear that the shorter mRNAs were more likely to be retrotransposed and to form processed pseudogenes or pseudogene fragments; the longer functional proteins, certainly their corresponding mRNAs, were less likely to be inserted into genomes by retrotransposition. Even if they did get inserted, they would be lost during evolution of the genome because of their greater influence on the structure and function of the genome. It has been reported that when mRNA sequences are longer than a specific value, they can no longer be retrotransposed in genomes [35].

\subsection{The distribution of pseudogenes and pseudogene fragments within the chromosomes}

The distribution of the functional genes, pseudogenes and pseudogene fragments within each of the $P$. tricornutum chromosome was calculated (Figure S7). The results showed that the distribution of functional genes in each chromosome was more or less uniform, while no such rule could be applied to the distribution of the pseudogenes and pseudogene fragments in each chromosome.

\section{Conclusions}

Because the $P$. tricornutum genome was found to contain many functional genes derived from animals, plants and bacteria, the $P$. tricornutum genome has been reported to be more like an animal genome than a plant genome [32]. The method that has been used to identify pseudogenes in the human genome was optimized according to the features of $P$. tricornutum genome. Thus the method described in this paper was appropriately used to identify and analyze pseudogenes in the $P$. tricornutum genome. A total of 1654 processed pseudogenes, 714 duplicated pseudogenes, and 4729 pseudogene fragments that proved to be accurate and 
authentic were identified. As far as we know, this is the first pseudogene study on algae. The results should offer insights into the structure and evolution of the $P$. tricornutum genome.

This work was supported by the National Basic Projects for Science and Technology (SQ2012FY4910019-1), the Nonprofit Research Project for the State Oceanic Administration (201105008-2) and the National Natural Science Foundation of China (41176137).

1 Vanin E F. Processed pseudogenes: Characteristics and evolution. Annu Rev Genet, 1985, 19: 253-272

2 Mighell A J, Smith N R, Robinson P A, et al. Vertebrate pseudogenes. Febs Lett, 2000, 468: 109-114

3 Nowak M A, Boerlijst M C, Cooke J, et al. Evolution of genetic redundancy. Nature, 1997, 388: 167-171

4 Tachida H, Kuboyama T. Evolution of multigene families by gene duplication: A haploid model. Genetics, 1998, 149: 2147-2158

5 Force A, Lynch M, Pickett F B, et al. Preservation of duplicate genes by complementary, degenerative mutations. Genetics, 1999, 151: 1531-1545

6 Mccarrey J R, Thomas K. Human testis-specific PGK gene lacks introns and possesses characteristics of a processed gene. Nature, 1987, 326: 501-505

7 Glusman G, Yanai I, Rubin I, et al. The complete human olfactory subgenome. Genome Res, 2001, 11: 685-702

8 Balakirev E S, Ayala F J. Pseudogenes: Are they "Junk" or functional DNA? Annu Rev Genet, 2003, 37: 123-151

9 Hirotsune S, Yoshida N, Chen A, et al. An expressed pseudogene regulates the messenger-RNA stability of its homologous coding gene. Nature, 2003, 423: 91-96

10 Svensson O, Arvestad L, Lagergren J. Genome-wide survey for biologically functional pseudogenes. PLoS Comp Biol, 2006, 2: e46

11 Lerat E, Ochman H. Recognizing the pseudogenes in bacterial genomes. Nucleic Acids Res, 2005, 33: 3125-3132

12 Harrison P, Kumar A, Lan N, et al. A small reservoir of disabled ORFs in the yeast genome and its implications for the dynamics of proteome evolution1. J Mol Biol, 2002, 316: 409-419

13 Benovoy D, Drouin G. Processed pseudogenes, processed genes, and spontaneous mutations in the Arabidopsis genome. J Mol Evol, 2006, 62: $511-522$

14 Nelson D R, Schuler M A, Paquette S M, et al. Comparative genomics of rice and Arabidopsis. Analysis of 727 cytochrome P450 genes and pseudogenes from a monocot and a dicot. Plant Physiol, 2004, 135: 756-772

15 Fran Oise T N, Shu O, Robin B C. Identification and characterization of pseudogenes in the rice gene complement. BMC Genomics, 2009, 10: 317

16 Harrison P M, Echols N, Gerstein M B. Digging for dead genes: An analysis of the characteristics of the pseudogene population in the Caenorhabditis elegans genome. Nucleic Acids Res, 2001, 29: 818-830

17 Harrison P M, Milburn D, Zhang Z, et al. Identification of pseudogenes in the Drosophila melanogaster genome. Nucleic Acids Res, 2003, 31: 1033-1037

18 Khelifi A, Duret L, Mouchiroud D. HOPPSIGEN: A database of human and mouse processed pseudogenes. Nucleic Acids Res, 2005, 33(Database issue): D59-D66

19 Zhang Z, Harrison P M, Liu Y, et al. Millions of years of evolution preserved: a comprehensive catalog of the processed pseudogenes in the human genome. Genome Res, 2003, 13: 2541-2558

20 Torrents D, Suyama M, Zdobnov E, et al. A genome-wide survey of human pseudogenes. Genome Res, 2003, 13: 2559-2567

21 Lander E S, Linton L M, Birren B, et al. Initial sequencing and analysis of the human genome. Nature, 2001, 409: 860-921

22 Yeh R F, Lim L P, Burge C B. Computational inference of homologous gene structures in the human genome. Genome Res, 2001, 11: 803-816

23 Field C, Behrenfeld M, Randerson J, et al. Primary production of the biosphere: Integrating terrestrial and oceanic components. Science, 1998, 281: 237

24 Falkowski P, Barber R, Smetacek V. Biogeochemical controls and feedbacks on ocean primary production. Science, 1998, 281: 200

25 Treguer P, Nelson D, Van Bennekom A, et al. The silica balance in the world ocean: A reestimate. Science, 1995, 268: 375

26 Werner D. The Biology of Diatoms: Silicate Metabolis. Berkeley: University of California Press, 1977. 149

27 Gibbs S. The chloroplasts of some algal groups may have evolved from endosymbiotic eukaryotic algae. Ann Ny Acad Sci, 1981, 361: 193-208

28 Delwiche C, Palmer J. The origin of plastids and their spread via secondary symbiosis. Plant System Evol Suppl, 1997, 11: 53-86

29 Medlin L K, Kooistra W, Schmid A M M. The origin and early evolution of the diatoms: Fossil, molecular and biogeographical approaches. Cracow, Poland: W. Szafer Institute of Botany, Polish Academy of Sciences Press, 2000. 13-35

30 Mcfadden G, Van Dooren G. Evolution: Red algal genome affirms a common origin of all plastids. Curr Biol, 2004, 14: R514-R516

31 Nisbet R, Kilian O, Mcfadden G. Diatom genomics: Genetic acquisitions and mergers. Curr Biol, 2004, 14: 1048-1050

32 Bowler C, Allen A E, Badger J H, et al. The Phaeodactylum genome reveals the evolutionary history of diatom genomes. Nature, 2008, 456: $239-244$

33 Zhang Z, Harrison P, Gerstein M. Identification and analysis of over 2000 ribosomal protein pseudogenes in the human genome. Genome Res, 2002, 12: 1466-1482

34 Li W H, Gojobori T, Nei M. Pseudogenes as a paradigm of neutral evolution. Nature, 1981, 292: 237-239

35 Gon Alves I, Duret L, Mouchiroud D. Nature and structure of human genes that generate retropseudogenes. Genome Res, 2000, 10: $672-678$

Open Access This article is distributed under the terms of the Creative Commons Attribution License which permits any use, distribution, and reproduction in any medium, provided the original author(s) and source are credited. 